\title{
HOW ARE DOING? CAN WE DO BETTER? MOTHER AND BABY SEPARATION DUE TO HYPOGLYCAEMIA

\section{Background and Aims}

There is a strong evidence that separation of mother and baby soon after birth can have a profound effect on maternal mental health, breastfeeding, and long-term morbidity for mother and child. Babies born at 37-38 weeks gestation accounted for $21 \%$ of all term live births in England (as per NHS Improvement) of whom, 55\% were due to hypoglycaemia. Our project aims to reduce the admission rate of those babies to our neonatal unit (NNU) due to hypoglycaemia by at least $25 \%$ by May 2019, hence enhancing the mother-baby bonding relationship.

\section{Method}

A quality improvement project designed at Leeds Teaching Hospitals Trust (LTHT) using Life QI system and many PDSA cycles to identify and tackle the reasons for NNU admissions of babies due to hypoglycaemia.

\section{Results}

18-month data audited (01/2016-07/2017) and revealed that at least 46 babies were admitted to NNU and transitional care unit (TCU) primarily due to hypoglycaemia. Inclusion and exclusion criteria presented in Table 1. Demographics of these babies and interventions presented in Table 2. Hypoglycaemia was the main reason for admission. Temperature was thought to be a major factor and it is multifactorial requiring intervention on multiple levels. Action plan is set out (as per the driver diagram) in order to achieve our target, and family satisfaction survey has also been designed.

\section{Aim Primary Drivers Secondary Drivers Change Ideas}

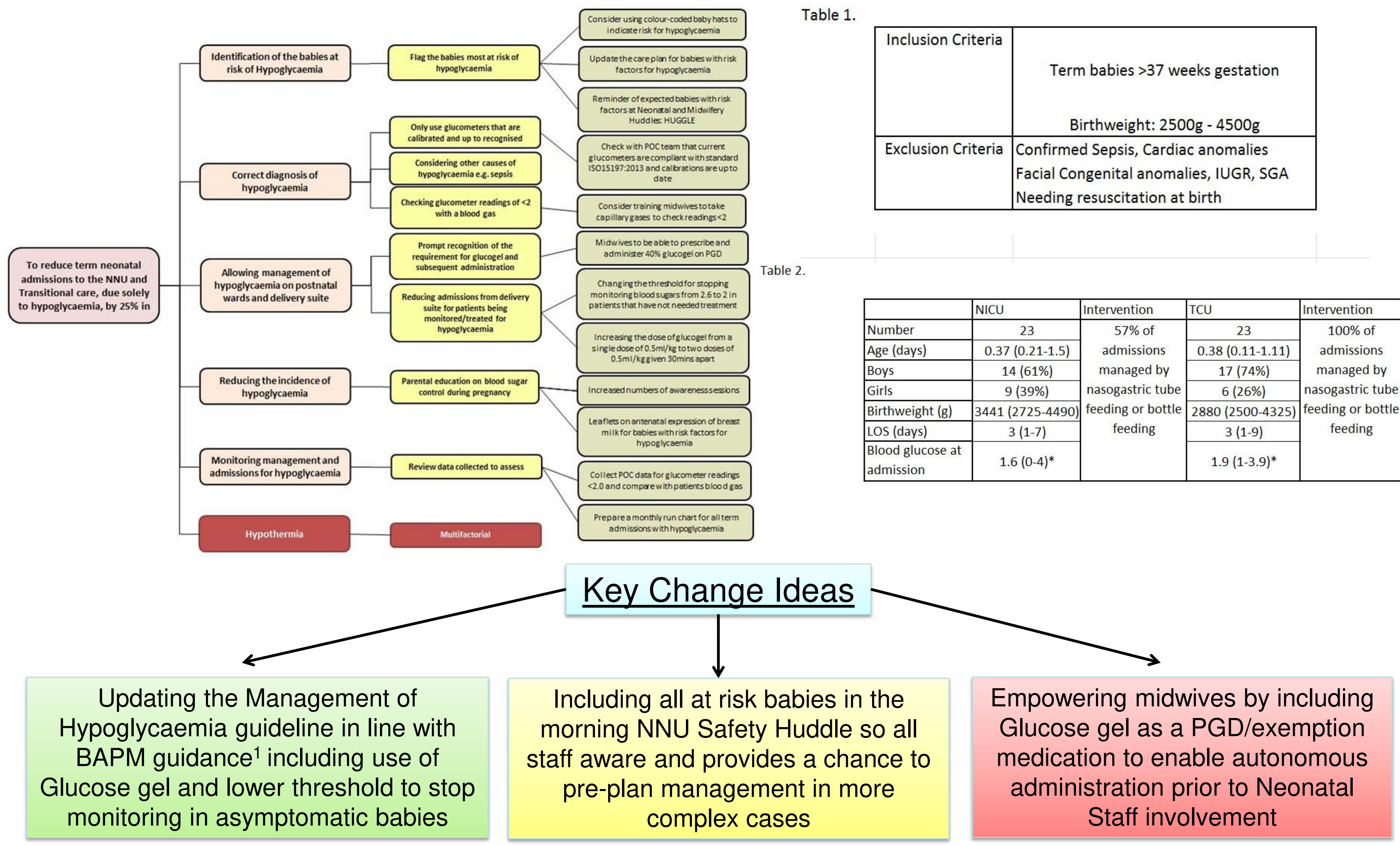

\section{Conclusion}

Separation of mother and baby due to hypoglycaemia could be avoided in many ways. Team collaboration and parental satisfaction are our drives. Prospective data collection is ongoing with weekly run charts tracking progress. Ongoing staff and parental education both with midwifery and neonatal teams to improve detection and prevention of admissions. Early data shows reduction in admission however complete data will be available at the end of first PDSA cycle in May 2019.

\section{References}

1. Identification and Management of Neonatal Hypoglycaemia in the Full Term Infant - A Framework for Practice (2017) : BAPM 